\title{
Slow Light at High Frequencies in an Amplifying Semiconductor Waveguide
}

\author{
Öhman, Filip; Yvind, Kresten; Mørk, Jesper
}

Published in:

Conference on Lasers and Electro-Optics and 2006 Quantum Electronics and Laser Science Conference. CLEO/QELS 2006.

Link to article, DOI:

10.1109/CLEO.2006.4627828

Publication date:

2006

Document Version

Publisher's PDF, also known as Version of record

Link back to DTU Orbit

Citation (APA):

Öhman, F., Yvind, K., \& Mørk, J. (2006). Slow Light at High Frequencies in an Amplifying Semiconductor Waveguide. In Conference on Lasers and Electro-Optics and 2006 Quantum Electronics and Laser Science Conference. CLEO/QELS 2006. (pp. 1-2). IEEE. https://doi.org/10.1109/CLEO.2006.4627828

\section{General rights}

Copyright and moral rights for the publications made accessible in the public portal are retained by the authors and/or other copyright owners and it is a condition of accessing publications that users recognise and abide by the legal requirements associated with these rights.

- Users may download and print one copy of any publication from the public portal for the purpose of private study or research.

- You may not further distribute the material or use it for any profit-making activity or commercial gain

- You may freely distribute the URL identifying the publication in the public portal 


\title{
Slow Light at High Frequencies in an Amplifying Semiconductor Waveguide
}

\author{
Filip Öhman, Kresten Yvind and Jesper Mørk \\ COM.DTU Department of Communications, Optics \& Materials, Nano.DTU, Technical University of Denmark \\ Build.345v,DK-2800 Kgs.Lyngby,Denmark,fo@com.dtu.dk
}

\begin{abstract}
We demonstrate slow-down of a modulated light signal in a semiconductor waveguide. Concatenated amplifying and absorbing sections simultaneously achieve both amplification and a controllable time delay at $15 \mathrm{GHz}$.

(C) 2005 Optical Society of America

OCIS codes: (250.5980) Semiconductor optical amplifiers, (070.6020) Signal processing
\end{abstract}

\section{Introduction}

The possibility of controlling the propagation speed of light has received a lot of attention lately [1-6]. The effect has been demonstrated using electromagnetically induced transparency (EIT) in ultracold atomic gases [1] as well as coherent population oscillation (CPO) in a ruby crystal [2] and semiconductor structures [3-6]. In this paper we investigate a waveguide device where several alternating sections of semiconductor optical amplifiers (SOA) and electro absorbers (EA) are used for enhancing the delay, and simultaneously providing net gain. The control of both phase and amplitude is important for applications in for example optically fed phased array antennas.

\section{The device}

The investigated device, shown in Fig. 1, consists of a single waveguide with a number of separate electrodes defining the amplifier and absorber sections, respectively. The waveguide is angled 7 degrees relative to the cleaved facets, which are also anti reflection coated to reduce reflections into the waveguide. The length of the SOA and EA sections are 545 and $120 \mu \mathrm{m}$, respectively. The active material consists of five $7.0 \mathrm{~nm}$ thick, compressively strained, InGaAsP quantum wells, in a strain compensated structure.
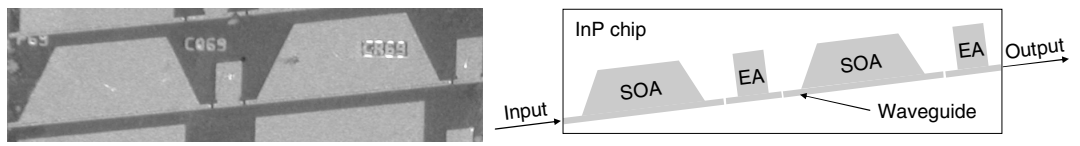

Fig. 1. Photo and schematic of the investigated device with alternating amplifiers (SOA) and saturable absorbers (EA).

The experimental setup and theoretical model

The experimental setup is shown in Fig. 2 and is based on the measuring technique of [2]. A network analyzer is used to generate a weak intensity modulated signal on a continuous wave laser beam. The signal is amplified and transmitted through the SOA-EA device. The phase delay is measured relative to a reference beam at low input power and measured at zero reverse bias of the absorber, i.e. where the delay is small. The input power is controlled by a variable attenuator and the polarization is set using a polarization controller. The model used is based on a fourwave mixing description of the device and was presented in detail in [5]. The description includes the double sideband modulation used in the experiment.

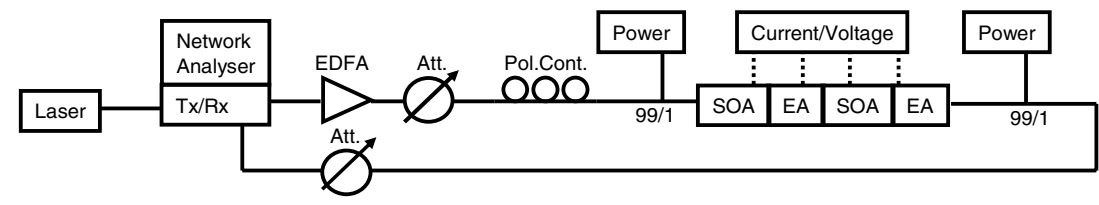

Fig. 2. The experimental setup used for the measurements.

\section{Results and discussion}

The phase shift and chip gain using both SOA-EA pairs of the device are shown in Fig. 3 (a) and (b). The first and second gain sections are biased at 80 and $30 \mathrm{~mA}$, respectively. This choice is made in order to optimize the device so that the two EAs give maximum phase delay at the same reverse bias. In 3 (a) the time delay corresponding to the phase shift and the modulation frequency, which is $15 \mathrm{GHz}$, is also displayed. The maximum phase shift of about 38 degrees corresponds to a change in the effective group index, and hence the propagation speed of the modulated 


\section{CMN1.pdf}

signal, by a factor of 3.6 assuming a background group index of 3.4. For an input power of about $0.5 \mathrm{dBm}$ the maximum delay coincides with zero $\mathrm{dB}$ gain in the chip, as seen in 3 (b). For higher input powers the SOAs are more saturated and the gain decreases but for lower powers both a tunable delay and net gain can be achieved.
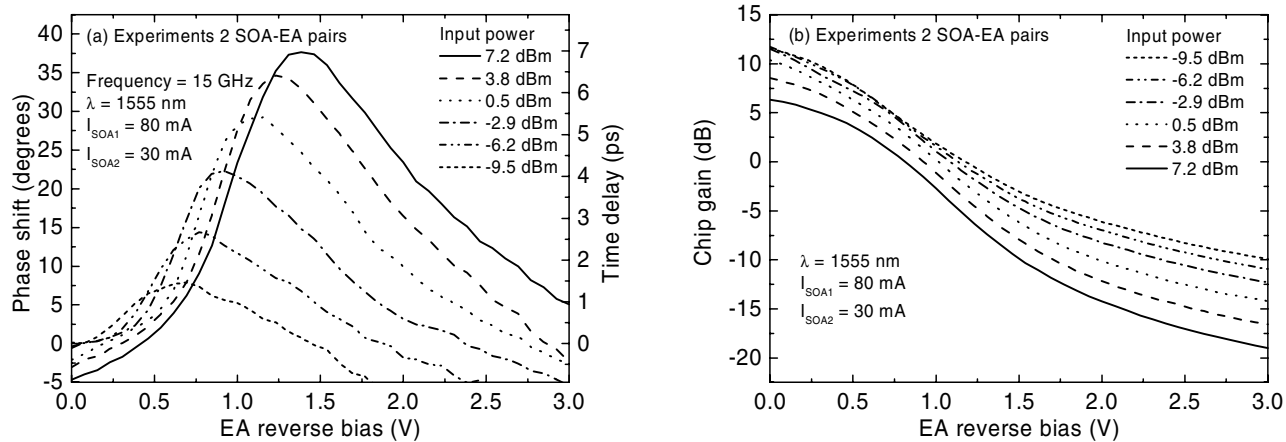

Fig. 3. Measured phase change and corresponding time delay (a) as well as chip gain (b) using both SOA-EA pairs of the device. The curves are plotted as function of the reverse bias of the two EA sections for different input powers.

In Fig. 4 the effect of cascading the SOA-EA sections is investigated by measurements on a single SOA-EA pair for different input power (a) and simulations of several pairs at fixed input power (b). In 4 (a) a similar situation as in Fig. 3 is shown except that the last two sections of the device are biased at transparency and do not contribute to the phase shift. The measured phase shift thus corresponds to the shift attained from one SOA-EA pair. Comparing Fig. 4 (a) and Fig. 3 (a) it is seen that the additional SOA-EA pair doubles the attainable delay for the low input power cases, but as the input power increases the increase in delay from the additional pair is less substantial. This can be attributed to the larger saturation of the SOA gain by the input power, which gives a negative delay, as seen most clearly at low reverse biases. Furthermore the reduced gain means that the power injected into the second EA is lower relative to the first EA, which also results in a smaller increase in delay when a second pair is added. In Fig. 4 (b) the theoretical model is used for investigating the phase shift for several SOA-EA pairs. The calculations agree well with the measurements. The phase shift, both positive from the EAs and negative from the SOA, increases with additional pairs. The simulations further indicate that an even larger phase shift can be attained by cascading several SOA-EA pairs.
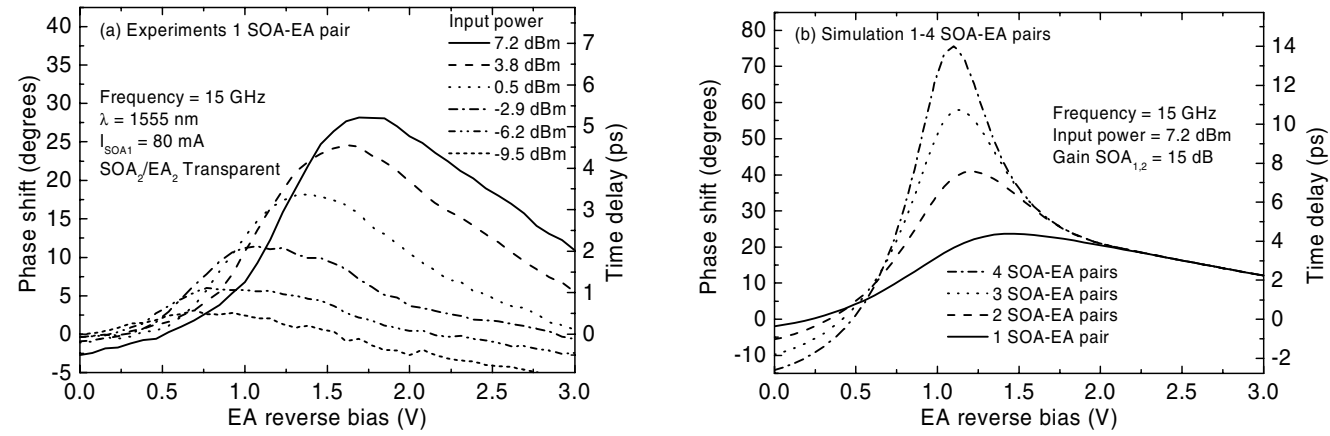

Fig. 4. Measured phase change and corresponding time delay using only the first SOA-EA pair of the device for different input powers (a) and calculations of the phase change and time delay in 1-4 SOA-EA pairs for fixed input power (b).

\section{Conclusions}

By combining gain and absorber sections in the same waveguide we have demonstrated slow light at a high modulation frequency $(15 \mathrm{GHz})$ in an amplifying device. By concatenating sections the phase shift can be scaled to high values, while allowing control of the amplitude. Simulations indicate that very large phase shifts are feasible.

\section{References}

[1] L.V. Hau et al. "Light speed reduction to 17 meters per second in an ultracold atomic gas", Nature 397, pp. 594-598 (1999).

[2] M. Bigelow et al. "Observation of ultraslow light propagation in a ruby crystal at room temperature", Phys. Rev. Lett. 90, 113903-1-4 (2003).

[3] C. J. Chang-Hasnain et al. "Variable optical buffer using slow light in semiconductor nanostructures", Proc. IEEE 91, pp. 1884-1897 (2003).

[4] P. Palinginis et al. "Ultra-slow light $(<200 \mathrm{~m} / \mathrm{s})$ propagation in a semiconductor nanostructure", Appl. Phys. Lett. 87, 171102 (2005.)

[5] J. Mørk et al. "Slow light in a semiconductor waveguide at gigahertz frequencies," Opt. Express vol. 13, no. 20 , pp. 8136-8145.

[6] M. v.d. Poel et al. "Controllable delay of ultrashort optical pulses in a semiconductor quantum dot amplifier" Opt. Express vol. 13, no. 20, pp. 8032-8037 (2005). 